\title{
Un système organisé pour la gestion de crise dans les vallées alpines
}

\author{
A well organized system for the crisis management in the Alpine valleys
}

\author{
par R. Raja \\ Région Lombardie, Italie \\ Directeur du Service de Protection Civile
}

This paper deals with the establishment of an integrated system of the "crisis management" from the flood forecasting (thanks to hydropluviometric control) to the alert of the local civil protection authorities, through the risk communication with the citizens, both in "time of peace" and during the events.

It will be experienced in an Alpine valley (north-west of the Come lake) a new system of real-time monitoring of the Livo river gauge. This system also measures the solid transport which is very important during summer rapid floods. This system links a new technology to a set of operation plans, alert procedures and information.

\section{I $\square$ LE PROBLÈME DES INONDATIONS DANS LES VALLÉES ALPINES}

L'un des problèmes plus aigus sur le territoire de la Région Lombardie est - surtout dans les dix dernières années - le risque hydrogéologique à tous les niveaux. Eboulements et inondations sont habituels : il suffit d'une pluie un peu plus intense que d'habitude, et parfois pas tellement, pour déchaîner des phénomènes d'inondations qui se traduisent, lors de la saison d'été, par un danger réel pour les habitants des nombreuses vallées alpines et les touristes qui les fréquentent.

Même sans arriver à la catastrophe de l'inondation en Valtelline de 1987 (trois provinces impliquées, plus de 40 morts, trente mille habitants évacués, vingts milliards de francs français de dommages), d'importants événements se produisent presque tous les ans en Lombardie : inondation du fleuve Olona (province de Varèse) en septembre 1995 ; des fleuves Brembo, Adda et Oglio en novembre 1996 ; en ValChiavenna et Haut-Lario (Lac de Côme) en juin 1997 ; la coulée de boue à Ardenno (province de Sondrio), en juin 1998 ; éboulements, coulées de détritus, d'une fréquence impressionnante, et aux conséquences surtout psychologiques.

Dans ces dernières années, en effet, la demande de sûreté de la population a augmenté énormément : à l'Etat, et également à la Région, les gens demandent une protection souvent impossible, incompatible avec leur désir (ou avec leur intérêt économique) de rester dans les zones définies "sous risque".
Ce qui n'a pas parallèlement augmenté, c'est le sens de la mesure et de l'à-propos, soit la conscience que l'habitat ne peut pas être "contre nature" : il n'est plus culturellement ni économiquement acceptable d'élever le niveau de protection des agglomérations (surtout à la montagne) au détriment de la conformation naturelle des lieux. On a scientifiquement démontré que l'élévation ou la consolidation des digues d'un fleuve, en augmentant la sensation de sécurité, détermine avec le temps un accroissement de l'occupation humaine dans les terrains historiquement sous risque. Cela implique également un accroissement de la vulnérabilité du lieu, et enfin une exceptionnelle élévation du risque au moment où - même après des dizaines d'années, quand la "mémoire historique" a été perdue - un événement de crue aussi exceptionnel entraîne tout ce qu'on a réalisé et détermine la catastrophe.

En tout cas, des études développés récemment par la Région Lombardie $^{1}$ ont calculé que dans les cinq derniers siècles sur le territoire régional on a eu 440 événements de crue significatifs, à l'exclusion des éboulements et des inondations moins importantes. Ce qui signifie en effet un événement par an.

Si l'on considère seulement les événements affectant la moitié du territoire régional, soit les plus désastreux même en termes économiques, on enregistre en Lombardie 20 inondations au cours des deux derniers siècles, soit en moyenne une tous les dix ans : ce n'est pas un hasard si la dernière inondation de ce niveau s'est produite en Valtelline (province de Sondrio) en 1987.

1. Dans le "premier programme de prévision et prévention de Protection Civiel". Région Lombardie, octobre 1998, par le Service de Protection Civile de la Région Lombardie. 
Encore une considération avant d'entrer au cœur du problème.

Les manifestations de crues, presque uniquement torrentielles (la dernière crue importante du Po remonte à 1953, et les dommages principaux furent en Vénétie et Emilie, pas en Lombardie), se produisent le plus souvent pendant les mois d'été : entre mi-juin et mi-septembre se concentrent $80 \%$ des phénomènes. En d'autres termes, le risque est maximum pour les agglomérations et encore plus pour les campings des vallées alpines juste dans la période de plus grande concentration touristique.

\section{II — L'EXPÉRIMENTATION SUR UN BASSIN-TYPE}

Le Service de Protection Civile de la Région Lombardie est en train de développer, depuis quelques années, une politique plus active de prévention du risque, dans le cadre d'un changement du système régional de protection civile, qui implique non seulement une plus forte efficacité dans la gestion de crise, mais aussi une plus grande capacité de prévision et surtout de prévision des désastres naturels.

En d'autres termes, on a développé tous les système de contrôle connus et considérés comme scientifiquement valables pour surveiller les points à plus haut risque hydrogéologique : la prévention du risque d'éboulement et d'inondation a été identifiée par la Région Lombardie comme priorité absolue dans l'action de "protection civile".

Le résultat le plus important obtenu par le Service de Protection Civile a été sans doute la rédaction du ler Programme Régional de Prévision et Prévention de Protection Civile, instrument fondamental de connaissance de l'état du territoire et de la gravité de ses risques.

Du 1996 au 1998, les cinq risques principaux du territoire lombard ont été étudiés : hydrogéologique (éboulements, inondations), sismique, industriel, incendies de forêts, nucléaire. L'effort de synthèse scientifique-opérationnelle coordonné par le Service a impliqué 8 Directions Générales régionales, 15 experts dans l'équipe projectuelle, 4 Universités et Centres de Recherche pour produire une relation définitive, 12 cartes tématiques et un CD-ROM, mais surtout, au cours de l'activité, un ensemble d'accords, conventions avec des Instituts ou des Agences de recherche et surveillance du territoire.

De plus, l'une des avancées les plus significatives faites pendant l'élaboration du Programme a été l'institution du Service Météorologique Régional, au moyen d'une convention avec le centre de prévision le plus accrédité sur le territoire lombard, l'Ersal (Agence Régionale de Développement Agricole). Cela a permis au Service de Protection Civile de développer, entre autres choses, un ensemble de systèmes d'alerte des Préfectures pour le risque d'inondation, fondé sur la prévision de la quantité de pluie dans les $36-48$ heures suivantes, dans les 7 zones climatiques et dans les 11 provinces qui constituent la Lombardie.

Malgré ces résultats et la récente acquisition du radar météo de Spino d'Adda (province de Crémone) capable de travailler sur le "nowcasting" de toute la partie en plaine, on est resté impuissant concernant deux phénomènes qui vont prendre de plus en plus d'importance et seront de plus en plus fréquents sur le territoire régional : les "flash floods" (les inondations rapides, sur les torrents des vallées préalpines), et les orages, qui sont - surtout dans les grandes agglomérations en été - de plus en plus soudains, concentrés et ravageurs, avec leur cortège d'inondations, grêle, risques pour la circulation.

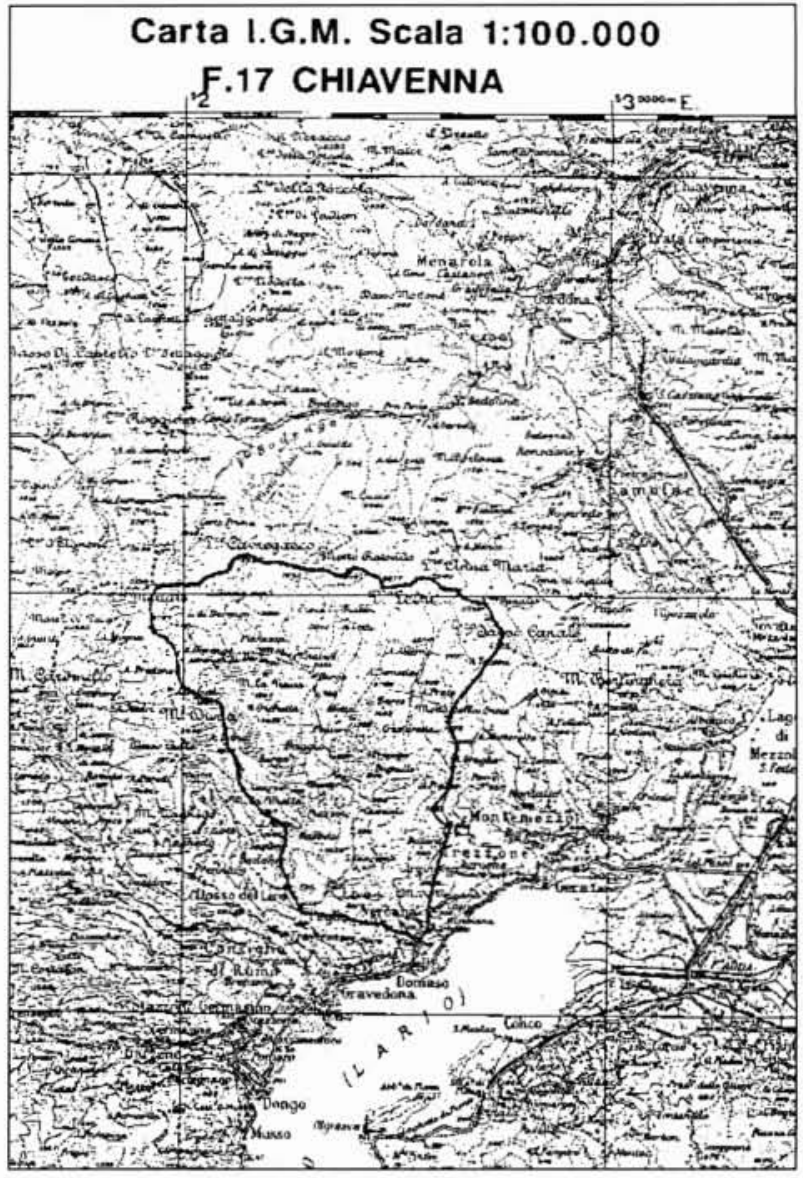

1. Carte IGM de la Région Lombardie.

Le Service de Protection Civile est en train de développer sur deux zones-échantillons, qui doivent servir d'exemple pour les vallées alpines lombardes, un système intégré de protection civile pour la prévention du risque d'inondation.

En particulier, l'attention se porte sur les "flash floods", c'est-à-dire les crues torrentielles soudaines, et donc les phénomènes où il ne s'agit pas seulement de surveiller les cours d'eau, mais aussi de prévoir le temps, et de surveiller en temps réel l'intensité des pluies.

Le premier bassin que l'on a choisi pour approfondir l'étude du phénomène "crue soudaine" liée au "transport en masse" de matériel alluvionnaire du haut de la montagne jusqu'au fond de la vallée, est celui du torrent Livo, sur le lac de Côme occidental, en province de Côme (Commune de Domaso).

Sur ce bassin (voir figure 1) on a chargé le CNR-IRPI de Turin ${ }^{2}$ d'étudier la répétition du phénomène des inondations et de projeter et mettre en place une station de surveillance avec des technologies innovatrices pour contrôler en temps réel, de la Salle d'Opérations du Service Protection Civile à Milan, la quantité de pluie, l'intensité des précipitations, le niveau de l'eau dans le torrent Livo, et surtout l'accroissement de la quantité du matériel lithoïde (pierres et terre de l'affouillement des bords) transporté par la crue du Livo (voir figure 2).

2. CNR-IRPI, soit Conseil National des Recherches, Institut de recherche pour la protection hydrogéologique ; il s'agit d'un Institut de Recherche publique, dépendant du ministère de la Recherche scientifique, avec siège à Turin. Il s'occupe de recherches sur le risque hydrogéologique sur tout le bassin du Po, du Piémont à la Vénétie. 

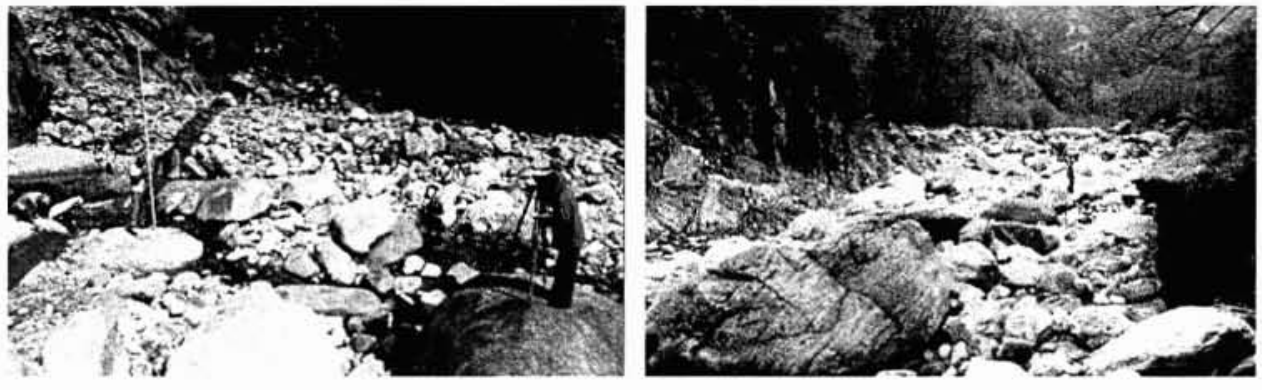

2. Lit du torrent Livo à peine en aval de la localité de Crotto Dangri (Commune de Livo). Exécution des relevés topographiques dans le site choisi pour l'évaluation du transport solide. A droite, lit du torrent Livo à la hauteur des refuges de montagne Dangri. On observe les dimensions parfois colossales des roches déposées.
L'étude est actuellement (septembre 1999) dans la phase de l'installation des premières et plus simples stations de surveillance météo-hydropluviométrique qui nous guideront ensuite dans la localisation et l'installation de la station-type pour la surveillance du transport en masse.

L'autre bassin où l'on va achever la seconde partie de l'expérimentation de l'efficacité du "système intégré" est celui du fleuve Brembo, affluent de l'Adda, en province de Bergame : la Haute Vallée Brembana. Ici la Région a stimulé la Communauté de Montagne de la Vallée Brembana, un consortium de 38 communes qui donnent sur la partie du fleuve Brembo de la source dans les Préalpes Orobies jusqu'au nord de la ville de Bergame, à élaborer le Plan d'Urgence inter-communale pour le risque hydrogéologique, c'est-à-dire les éboulements, inondations, avalanches.

Il vaut la peine de rappeler que même la Haute Vallée Brembana fut impliquée dans la catastrophe de Valtelline en 1987, avec une crue du fleuve Brembo dévastatrice.

La rédaction du Plan est actuellement en cours, et devait s'achever fin novembre 1999. Le concept-guide du Plan est : de la surveillance au préavis, à la prévention fondée sur la connaissance des phénomènes, la conscience des scénarios de risque, le soin des procédures, l'habitude avec les règles de conduite pour la population.

\section{III — LE SYSTÈME INTÉGRÉ DE PRÉVENTION DU RISQUE}

Revenons au bassin du torrent Livo, choisi pour l'étude sur l'applicabilité du système à la situation-type des vallées alpines-préalpines : il s'agit d'un bassin de près de $50 \mathrm{~km}^{2}$. articulé dans un filet hydrographique à mailles serrées, avec 4 sous-bassins, et une amplitude altimétrique qui va des $2534 \mathrm{~m}$ du Pizzo Cavregasco aux $210 \mathrm{~m}$ du niveau de sortie sur le lac de Côme en Commune de Domaso.

Dans le bassin, le CNR-IRPI a recensé 19 événements de crue significatifs dans les 200 dernières années, dont dix dans 50 dernières années. On a vérifié aussi un accroissement de la fréquence des crues dans le dernier siècle, avec une tendance actuelle d'un événement tous les 4-5 ans. D'importants travaux de régulation du torrent et de protection hydraulique furent faits après la crue catastrophique ( 9 morts) du 8 août 1951, mais l'on estime en tout cas que les crues de ce niveau-là ont une période de retour de plus de 30 ans : la dernière s'est produite en septembre 1983, mais elle $n$ 'a pas fait de victimes.

L'aspect le plus significatif du risque existant sur le territoire est la situation de l'agglomération de la commune de Domaso (voir figure 3), placée exactement sur le cône de déjections à la sortie du torrent Livo dans le lac de Côme, avec une grande quantité de campings sur le terrain sous risque majeur, celui plus plat en bordure gauche du torrent. Ici le danger vient de l'inondation du lac, mais surtout du fait que ce terrain-là est historiquement "à disposition" des crues du torrent Livo, qui y aurait son débouché naturel en projection rectiligne de son cours. Ce n'est pas un hasard si l'expansion de l'agglomération de la localité de La Poncia, au sud de la route nationale côtière, a été minimale, et seulement dans les années 70 . alors que les établissements plus importants sont les campings, remplis de monde surtout en été, quand le danger est maximum (la répétition des phénomènes est à son apogée en septembre, juillet et août). Cependant, même le reste de l'agglomération, qui a vu sa population augmenter de $40 \%$ depuis 1876 . justement sur la rive droite du
3. Accroissement de la superficie urbanisée dans le cône d'alluvions du Livo

(commune de Domaso). Période 1876-1985.

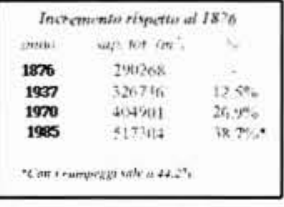



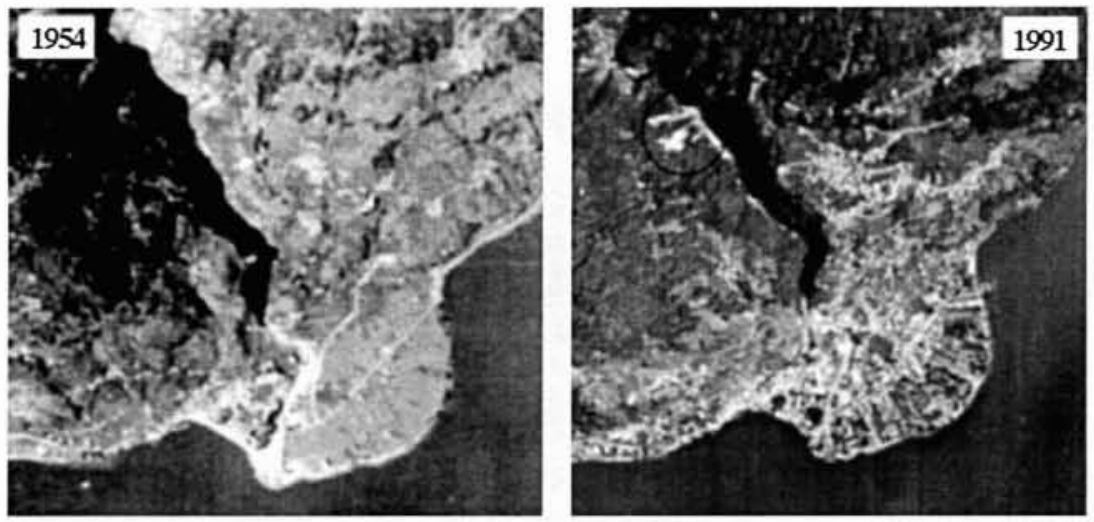

4. Tracé terminal du torrent Livo dans la commune de Domaso. La comparaison entre les deux photographies aériennes représentées $(5 / 5 / 78$ et $2 / 4 / 92)$ montre les dimensions anormales prises par le torrent trois ans après la crue catastrophique du 8 août 1951 dans un contexte d'utilisation de la seule zone restée inchangée par rapport au siècle précédent. Par contre, dans les conditions actuelles, le canal d'écoulement, défini en partie par les structures artificielles, est à peine visible. Une large partie du champ d'expansion du cours d'eau à gauche s'est urbanisée, avec une augmentation des espaces occupés nettement supérieure à $40 \%$ par rapport à 1876 . En cas de répétition d'un événement extrême, les conditions de risque géomorphologique et hydraulique pourraient aujourd'hui s'aggraver par l'accroissement de la réserve de matériaux solides dans le torrent en amont des zones habitées à la suite des deux éboulements survenus en 1977-78 bien visibles à droite.

Livo, dans la partie historiquement plus critique, est à risque, et exige une action urgente de prévention (voir image n.4).

La recherche actuellement en cours, comme l'on a déjà dit, tend à trouver la position géographiquement la plus appropriée pour la mise en place d'une station de surveillance-type pour la mesure du transport en masse ("debris flow"). Dès que l'on recevra des données valables, on pourra établir des seuils de risque, au franchissement desquels une alerte sera donnée, impliquant une évacuation ordonnée et contrôlée des agglomérations impliquées.

La séquence de l'étude-système intégré, conduite non seulement par le CNR-IRPI, mais aussi par les structures techniques intérieures de la Région Lombardie, sera donc : a) mise en place de la station-type de relevé (pluviomètre, hydromètre, volume et vitesse du transport) ;

b) analyse des données et comparaison avec les modèles de prévision de la pluie actuellement produits par le Service Météorologique Régional, pour la validation ;

c) comparaison des données reçues avec les phénomènes réels de crues, même réduites, ou d'éboulements, pour estimer une série de seuils au-delà desquels les phénomènes commencent à être irrévocablement désastreux ;

d) en correspondance de ces seuils, définition des "codes d'alerte", sur les modèles actuellement en vigueur sur le reste du territoire lombard, par ex. "préalarme", "alarme", "urgence", pour indiquer trois niveaux, du plus bas au plus haut degré d'intensité des phénomènes, et donc de l'état de vigilance des structures opérationnelles de la protection civile (sapeurs-pompiers, police, personnel sanitaire d'urgence, techniciens des communes, volontaires) ;

e) définition des "scénarios de risque" plus probables correspondant du moins à deux niveaux-types de gravité des phénomènes, avec la cartographie des zones affectées, et la description des conséquences les plus probables, en termes de quantité et qualité de population et des biens impliqués, structures publiques, infrastructures, systèmes de transport, réseaux hydriques, égouts, services, etc. ;

f) définition d'un Plan d'Urgence décrivant les procédures d'alerte et d'évacuation, ainsi que d'autres mesures de protection, liées aux niveaux d'alarme correspondant aux différents seuils, et aux différents scénarios de risque ; le Plan indique ainsi tous les acteurs qui doivent agir sur la scène avant, pendant et après l'urgence, en établissant des règles de conduite et rôles, en indiquant dans une matrice "actions/responsabilités" : les actions qui doivent être accomplies, dans l'ordre, qui doit les accomplir ; on distingue le "responsable" (R) de l'action et celui qui doit apporter son "soutien" (S) ainsi que celui qui doit seulement être "informé" (I) de ce qui se passe à ce moment-là ; g) définition d'une stratégie de communication, qui doit impliquer et rendre responsable la population résidente (et informer aussi les touristes) du risque existant, et les rassurer sur le "niveau de risque acceptable" où l'on peut réellement achever une action de protection.

L'ensemble de toutes ces phases constitue un "système intégré de protection civile", qui d'un côté sert à stimuler les Autorités (Région, Provinces, Communes) à réaliser une vraie politique de travaux de prévention qui ne bouleverse pas le territoire, respecte la nature des lieux et tienne compte de l'histoire et de la répétition des désastres naturels ; d'un autre côté, il constitue le squelette d'une action de protection à court terme qui peut servir à sauver des vies, et des biens, dans une certaine limite. Finalement, il peut constituer un point de repère pour les actions coordonnées entre plusieurs acteurs, ce qui est un problème typique de la protection civile sous toute latitude.

\section{COMMUNICATION ET CULTURE D'AUTO-PROTECTION}

Au-delà de toute considération scientifique et technique, il vaut la peine de souligner qu'aucune action de protection civile ne peut être efficace, dans un pays démocratique, sans la pleine et consciente collaboration de la population et, en général, de celle qui est définie "opinion publique".

L'étude qu'on est en train de mener en Vallée Brembana, séparée pour des raisons "scientifiques" de l'étude en cours sur le bassin du torrent Livo, tend à vérifier quelle pourrait être la participation réelle de la population à un Plan d'Urgence qui, dans cette première version, est réalisé par la Communauté Montaine sous l'autorité de la Région Lombardie (Service de Protection Civile et Service de Géologie).

En d'autres termes, il faudra évaluer et expérimenter dans quelle mesure la connaissance approfondie des phénomènes possibles aura une incidence sur la conscience des citoyens, dans quelle mesure elle les poussera à se faire impliquer dans la mise à point des procédures opérationnelles, dans quelle mesure elle les aidera à comprendre où et comment 
orienter (ou pas) l'expansion de l'agglomération. Il est évident que le Plan ne peut pas fonctionner si la population ne connaît pas les procédures qui y sont définies : une évacuation réussit si les gens sont informés et ne sont pas bouleversés par le stress de l'urgence.

Mais c'est certainement ici le pas le plus difficile à franchir, car la population tend à refuser ou sous-estimer le risque, au début, mais - avec la conscience d'habiter un terrain à risque - l'appréhension augmente et l'on entre dans une phase de surestimation du risque. Enfin, c'est la pression sur les Autorités qui va augmenter, pour qu'on élève le niveau de protection par des œuvres structurelles permanentes : la politique traditionnelle des "travaux publics". Ou bien, on demande une sécurité absolue autant qu'impossible à atteindre.

A cet égard, le Service de Protection Civile régional a essayé de convaincre les maires d'être les vrais et premiers acteurs de la protection civile. En Italie c'est d'abord la loi qui rend les maires responsables de toute action de protection civile sur leur territoire, mais ça ne suffit pas à rendre les maires capables de travailler dans ce sens.

Alors on a cherché à instaurer des cours de formation, spécifiques sur tous les thèmes de protection civile, pour les maires des communes les plus menacées par le risque hydrogéologique dans les onze provinces de la Lombardie, et (on a mis en place actuellement 4 cours, et l'on continue) on prévoit de compléter les cours pour l'an 2000.

Il revient donc au maire d'abord, d'impliquer sa population dans la rédaction du Plan d'Urgence, de transformer cet instrument "bureaucratique" en une occasion de participation démocratique à la vie de la communauté, de connaissance de son propre territoire, et d'acceptation d'un niveau de risque compatible avec la conservation de la vie et de la propriété, mais aussi, en modifiant son attitude vers la nature et le paysage, d'acceptation de règles d'aménagement du territoire capables de réduire à sa racine le risque qu'un phénomène naturel comme l'inondation d'un torrent de montagne se traduise en un désastre en termes économiques et, surtout, en termes de pertes de vies humaines.

Les figures 1, 2, 3, 4 sont dues à la courtoisie de CNRIRPI de Turin, et appartiennent à l'étude actuellement en cours que cet Institut est en train de conduire pour la Région Lombardie. 